\title{
A NEW SPATIALLY ADAPTIVE TV REGULARIZATION FOR DIGITAL BREAST TOMOSYNTHESIS
}

\author{
Maissa Sghaier ${ }^{\star \dagger}$, Emilie Chouzenoux ${ }^{\star}$, Jean-Christophe Pesquet ${ }^{\star}$, and Serge Muller ${ }^{\dagger}$

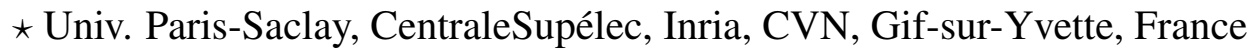 \\ $\dagger$ General Electrics Healthcare, Buc, France
}

\begin{abstract}
Digital breast tomosynthesis images provide volumetric morphological information of the breast helping physicians to detect malign lesions. In this work, we propose a new spatially adaptive total variation (SATV) regularization function allowing to preserve adequately the shape of small objects such as microcalcifications while ensuring a high quality restoration of the background tissues. First, an original formulation for the weighted gradient field is introduced, that efficiently incorporates prior knowledge on the location of small objects. Then, we derive our SATV regularization, and integrate it in a novel 3D reconstruction approach for DBT. Experimental results carried out on both phantom and clinical data show the great interest of our method for the recovery of DBT volumes showing small lesions.
\end{abstract}

Index Terms - Digital Breast Tomosynthesis, total variation, spatially adaptive regularization, 3D image reconstruction, optimization.

\section{INTRODUCTION}

In a will to reduce the mortality associated with breast cancer, heavy demands on the improvement of medical imaging modalities for breast cancer detection have been carried out in the last few years. Recently, Digital Breast Tomosynthesis (DBT) has demonstrated its superiority over X-ray mammography, the current modality of choice for breast cancer screening, for detecting lesions with comparable X-ray dose and a higher detection rate [1].

One of the most important features of DBT is that it provides a quasi-3D image of the breast. This is of a crucial importance, especially for young women with heterogeneous breasts, from which cancer detection can be difficult when using standard mammography. In an analog way that it is a difficult task to see a needle in a haystack, detecting by visual inspection a cancer lesion in a standard 2D mammography remains quite challenging. Yet, three-dimensional DBT provides a piercing view through the haystack, i.e., the breast, by reducing the impact of tissue structures overlapping [2] and so potentially increases the sensitivity and specificity of cancer detection. A key ingredient to guarantee such benefits is the image reconstruction algorithm. Exhaustive investigations and comparative studies on DBT reconstruction algorithms have clearly shown the advantages of iterative reconstruction algorithms [3]. Many of these algorithms rely on a convex optimization formulation employing a total variation (TV) regularization function [4, 5, 6]. The TV regularizer has illustrated its great performance in various applications in image processing [7, 8, 9]. However, the undesired staircasing side-effect of TV [10] has led researchers to propose alternative TV-based penalties, by proposing various smooth approximations [11, 12, 13, 14], by investigating the non-locality [15], or by computing a posterior mean instead of the MAP estimation framework [16]. Better recovery of anisotropic objects can also be reached, using anisotropic TV-based regularization [17, 18, 19, 20, 21]. Nonetheless, to the best of our knowledge none of them have provided a TV formulation that takes into consideration the heterogeneity (in shape and texture) within the image to be regularized. Breasts consist of different anatomical components such as tissues (background) and lesions that require a more sophisticated regularization when reconstructing the DBT volume. In such challenging context, our contribution is to propose a new Spatially Adaptive TV (SATV) regularization function which responds to the need for heterogeneous spatial regularization in DBT reconstruction, and accounts for the different morphological contents of the breast. Henceforth, we provide a new definition of the gradient field in the discrete image that takes into consideration an input detection map incorporating prior knowledge on the structural contents of the image. Thus, the SATV operator acts differently according to the sought local information around each voxel.

The paper is organized as follows: Section 2 provides the description of our convex optimization framework for DBT reconstruction. In Section 3, we present our main contribution which lies in the mathematical formulation of the SATV regularization function, and we discuss its incorporation in $3 \mathrm{D}$ reconstruction algorithms. Section 4 shows the experimental results carried out on both physical phantom and clinical data and discusses the obtained qualitative results. Finally, conclusions are drawn in Section 5 . 


\section{CLINICAL TASK-BASED RECONSTRUCTION IN DBT}

From a mathematical point of view, the reconstruction of the volumic image from DBT measurements is an inverse problem. The forward model is often simplified into the following linear form [3]:

$$
p=A d+e
$$

where $p \in \mathbb{R}^{n}$ is a vector derived from the acquired projections, $d \in \mathbb{R}^{m}$ is a vector representing the unknown volume, $A \in \mathbb{R}^{n \times m}$ is a matrix describing the geometry of the system, and $e \in \mathbb{R}^{n}$ is the noise. The objective is then to have an accurate reconstruction $d$ from under-sampled data $p$. Since the sampling rate for acquiring the projections $p$ is very low in standard DBT configurations, it is challenging to obtain a solution $d$ which reaches a globally satisfactory visual quality. It appears also crucial to take into consideration, in the reconstruction process, the different clinical tasks that are carried out by the radiologist when reading DBT images, in particular microcalcifications detection. To reach both of these goals, we formulate the solution to (1) as the minimization of a penalized least squares cost function:

$$
\underset{d \in\left[0, d_{\max }\right]^{m}}{\operatorname{minimize}} \frac{1}{2}\|p-A d\|_{2}^{2}+\Phi(d)-D(d) .
$$

Hereabove, $\Phi: \mathbb{R}^{m} \rightarrow \mathbb{R}$ is a TV-based spatial regularizer, and $D: \mathbb{R}^{m} \rightarrow \mathbb{R}$ denotes a detectability function enhancing the visibility of certain objects related to the sought clinical task. In a previously published work we have shown that the minimization of the cost function (2) yields good results in terms of microcalcifications enhancement in DBT reconstruction [22] while a slight spreading on the microcalcifications shapes can be observed. Furthermore, it can be expected that a better image quality trade-off is obtained by designing a TVbased regularization taking into account some prior knowledge on the different regions of the volume, as it is already done for building the clinical-based term $D$. Subsequently, we introduce a new total variation operator that enables an apter spatial regularization in the DBT volume accounting for the sought morphological content in the neighborhood of each voxel.

\section{SATV REGULARIZATION}

\subsection{Mathematical formulation}

With the aim to make regularization adaptive to the local contents of the DBT volume $d \in \mathbb{R}^{m}$, we define the new SATV regularization function as follows

$$
\Phi(d)=\sum_{i=1}^{m} \psi\left((1-\eta) \lambda_{i}(\Delta d)_{i}+\eta\left(1-\lambda_{i}\right) \delta d_{i}\right)
$$

where $\delta=\left[\begin{array}{lll}1 & 1 & 1\end{array}\right]^{\top}, \psi: \mathbb{R}^{3} \rightarrow \mathbb{R}$ represents a sparsity promoting function which applies either on the image intensity or its gradients, $\eta$ controls the overall contribution of each regularizing term, $\Delta$ denotes the $3 \mathrm{D}$ gradient operator that is expressed as

$$
\left(\Delta_{i}\right)^{\top}=\left[\begin{array}{lll}
\Delta_{i}^{x} & \Delta_{i}^{y} & \Delta_{i}^{z}
\end{array}\right]^{\top}
$$

where $\Delta_{i}^{x}$ (resp. $\left(\Delta_{i}^{y}\right)$ and $\left(\Delta_{i}^{z}\right)$ ) denotes the horizontal, (resp. vertical and depth) gradient at voxel $i \in\{1, \ldots, m\}$, with zero-boundaries assumption. It must be emphasized that the nonnegative weight parameters $\left(\lambda_{i}\right)_{1 \leq i \leq m}$ play a crucial role in the adaptation to the local spatial contents which is performed. In particular when, for every $i \in\{1, \ldots, m\}, \lambda_{i}=0$ and $\psi=\|\cdot\|_{1}, \Phi$ reduces to the $\ell_{1}$ regularization, whereas, when for every $i \in\{1, \ldots, m\}, \lambda_{i}=1$ and $\psi=\|\cdot\|$, we recover the standard isotropic total variation. In the case of DBT volumes, we would like to apply different regularizations in the areas where microcalcification are present/absent. The advantage of formulation (2) is that it allows us to exploit the detection map, extracted from a Computer-Aided Detection like tool [23], that enables the retrieval of the location of microcalcifications in the DBT volume. Using this detection map, a prior estimate $\mu_{i}$ of the normalized intensity value of voxel $i \in\{1, \ldots, m\}$ can be derived [22]. We recall that the microcalcifications are defined, in this framework, as localized structures lying within a predefined radius range. Henceforth, the proposed expression of the weight parameters is given by

$$
\lambda_{i}= \begin{cases}1 & \text { if } \mu_{i} \leq \nu-\theta \\ \frac{\theta+\nu-\mu_{i}}{2 \theta} & \text { if } \nu-\theta \leq \mu_{i} \leq \nu+\theta \\ 0 & \text { otherwise }\end{cases}
$$

where $\nu \in[0,+\infty[$ with $\theta \in] 0, \min (\nu, 1-\nu)]$. The value $\mu_{i}$ is high in zones where structures are present, while it is low in other areas. The threshold $\nu$ allows us to set the binary transition between the two zones, while the parameter $\theta$ is used to smooth the latter transition.

\subsection{Reconstruction algorithms}

In this section, we briefly explain how Problem (2) can be numerically handled when SATV regularization is used. From (3), function $\Phi$ can be reexpressed as

$$
\forall d \in \mathbb{R}^{m}, \quad \Phi(d)=\Psi(L d)
$$

where

$$
\forall u=\left(u_{i}\right)_{1 \leq i \leq m} \in\left(\mathbb{R}^{3}\right)^{m}, \quad \Psi(u)=\sum_{i=1}^{m} \psi\left(u_{i}\right),
$$

and

$$
L=(1-\eta)(\Lambda \otimes \delta) \Delta+\eta(1-\Lambda) \otimes \delta \in \mathbb{R}^{3 m \times m},
$$


where $\otimes$ denotes the Kronecker product and $\Lambda=$ $\operatorname{Diag}\left(\lambda_{1}, \ldots, \lambda_{m}\right)$. The function $\psi$ can be assumed proper, lower-semicontinuous and convex and the detectability function $D$ can be chosen linear [22]. Since $\Phi$ is the composition of matrix $L$ and the proper, lower-semicontinuous and convex function $\Psi$, a variety of splitting iterative algorithms can be applied to solve Problem (2):

- If the proximity operator of $\psi$ has a closed form expression, proximal algorithms [24] which include ADMM [25] and primal-dual methods [26] provide efficient solutions.

- If $\psi$ is Lipschitz-differentiable, then the problem can even be solved in a simpler manner through (possibly accelerated) projected gradient algorithms, where a projection onto the hypercube $\left[0, d_{\max }\right]^{m}$ is performed at each iteration.

\section{EXPERIMENTAL RESULTS}

In this section, we present experimental results carried out first on a physical phantom, then on two realistic clinical datasets. Each dataset contains 9 projections acquired using a DBT commercial system (Senographe Essential, GE Healthcare) with an angular aperture of $25^{\circ}$. The detector is composed of $3062 \times 2394$ detector elements of $100 \mu \mathrm{m}^{2}$ in size. The volumes are reconstructed under a $100 \mu \mathrm{m} \times 100 \mu \mathrm{m} \times 1 \mathrm{~mm}$ sampling grid. We aim at identifying small microcalcifications of size within the range $[0.2,0.4] \mathrm{mm}$ from the rest of the breast. Therefore, we rely on a map $\mu$ allowing us to detect structures with the latter size range following the approach proposed in [22]. We then construct $\left(\lambda_{i}\right)_{1 \leq i \leq m}$ as detailed in Section 3.1. The detection map is binarized by setting the threshold value $\nu$ so that the geometric shape of the detected microcalcifications are well recovered. Moreover, $\theta$ is manually finetuned to optimize the transitions between breast background and microcalcifications. Finally, we set $\eta$ in order to achieve a certain image quality optimization of DBT. In the general expression (3) of SATV regularization, a smooth approximation of the Euclidean norm is used as a penalty function, namely for every $u \in \mathbb{R}^{3}, \psi(u)=\beta \sqrt{\|u\|^{2}+\epsilon^{2}}$, where $(\beta, \epsilon) \in\left(\mathbb{R}_{+}\right)^{2}$ control the weight and smoothness of the regularization, respectively. The upper bound in the range value constraint is chosen equal to $d_{\max }=4095$. As explained in Section 3.2 the minimization of (2), in the considered setting, can be performed by projected gradient descent. The algorithm was initialized with the Filtered Back Projection solution, and ran until a maximum number of iterations (here equal to 200 ), which was shown to be sufficient to reach practical convergence in our experiments.

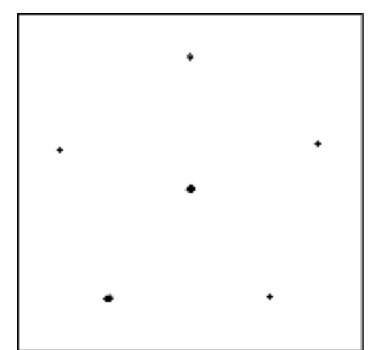

(a)

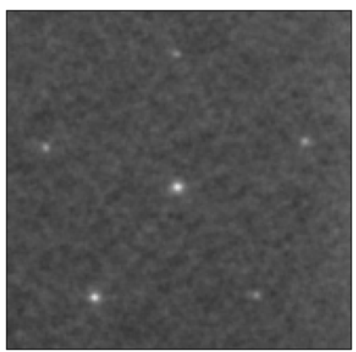

(c)

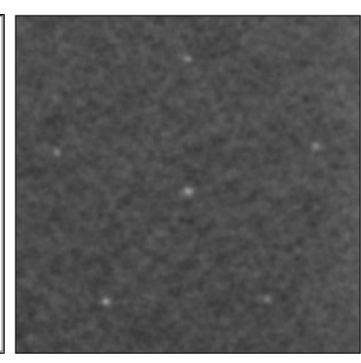

(b)

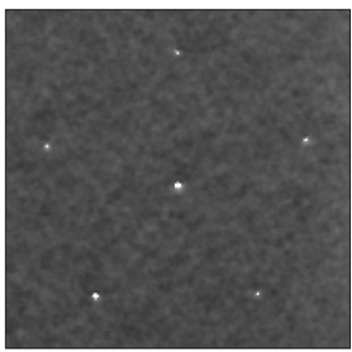

(d)
Fig. 1: Region of ACR phantom slice containing 6 ROIs: (a) Weights $\left(\lambda_{i}\right)_{1 \leq i \leq m}$, (b) DBT reconstruction with classical TV and no clinical term, (c) DBT reconstruction with classical TV and clinical term (d) DBT reconstruction with SATV regularization and clinical term.

\subsection{Physical phantom data}

We have first evaluated our approach using the physical phantom accreditation Model 015 (CIRS). Its dimension is $45 \times$ $102 \times 108 \mathrm{~mm}^{3}$, with uniform background. For the sake of visualization, we only display a zoomed region containing 6 microcalcifications of $0.3 \mathrm{~mm}$ in size and with known locations. We display in Fig. 1 a) the constructed $\left(\lambda_{i}\right)_{1 \leq i \leq m}$ as explained in Section 3.1. We set $\nu=1.5 \times 10^{-3}$ and $\theta=\nu / 3$. It is worth mentioning that the background is merely equal to zero in the detection map. Yet, the detected microcalcifications present different grey level values which will lead to a different regularization magnitude for each of them. The benefits of our proposed method as illustrated in Fig. 1(d) is twofold. First, compared to the results obtained with a classical DBT reconstruction (Fig. 1 b)), we significantly enhance the visibility as well as the sharpness of the detected microcalcifications. Second, compared to the results obtained with a clinical task-based reconstruction using a classical TV, as illustrated in Fig. 1.(c), we greatly improve the shape restoration of the enhanced microcalcifications. We emphasize that the comparison between the SATV-based reconstruction and the classical TV-based reconstruction is fair, since the regularization parameters, namely $\beta$ and $\eta$, are set such that the background is restored with the same quality. In this example, $\beta=600, \epsilon=10$ for classical TV while $\beta=630, \epsilon=10, \eta=0.048$ in the case of the new SATV regularization. 


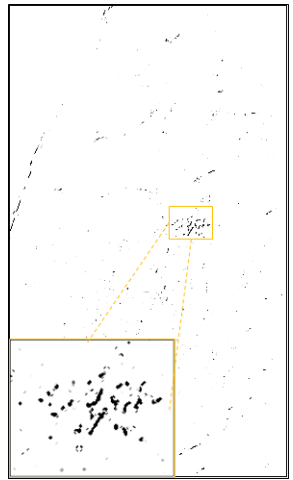

(a)

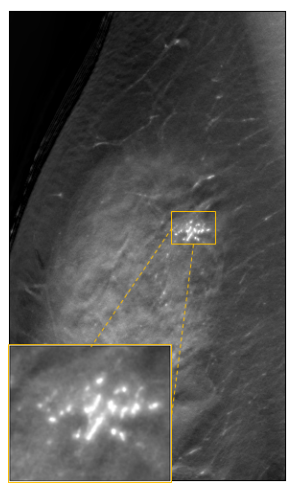

(c)

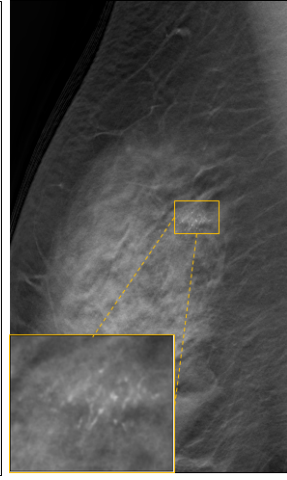

(b)

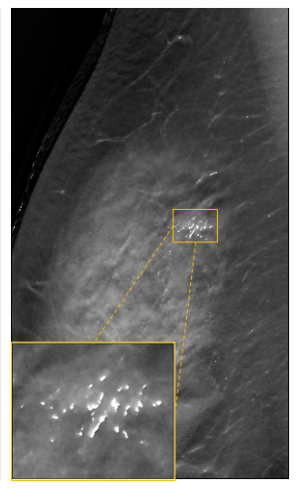

(d)
Fig. 2: Sample of slices of a DBT reconstruction with size $3062 \times$ $994 \times 67$ (pixels) : (a) Constructed $\left(\lambda_{i}\right)_{1 \leq i \leq m}$ from the representative clinical data. (b) DBT reconstruction with classical TV regularization and without the clinical term, (c) DBT reconstruction with classical TV regularization and the clinical term, (d) DBT reconstruction with SATV regularization and the clinical term.

\subsection{Clinical data}

We have also assessed the performance of our proposed approach on a clinical scenario. We considered here two $B I$ $R A D S b$ breast density clinical datasets, which correspond to breasts with scattered areas of fibroglandular density [27]. We show in Fig. 2 a case compromising a potentially malignant cluster of microcalcifications, while in Fig. 3 a case containing an isolated microcalcification. We display the map of $\left(\lambda_{i}\right)_{1 \leq i \leq m}$ obtained from the estimated signal where $\nu=$ 0.09 and $\theta=\nu / 3$, in Fig. 2 (a) and Fig. 3. a) respectively. Despite the presence of false positives in these latter maps, we note that the enhancement is more effective in the lesion area. One can clearly observe in Fig. 2 and in Fig. 3 the behavior already unveiled in the physical phantom dataset. With our proposed method, we highlight the advantage of simultaneously enabling a robust preservation of the shape of the enhanced microcalcifications and of a high quality restoration of the background tissues. Hereagain, the reconstruction with our proposed approach and the one based on classical TV provide

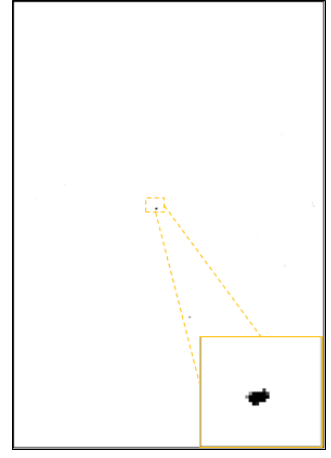

(a)

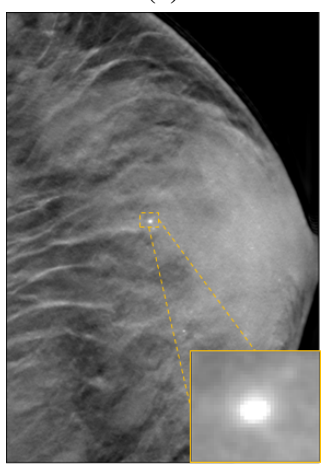

(c)

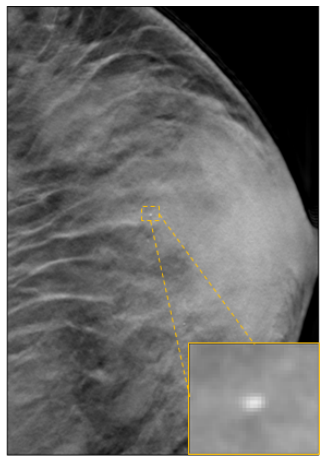

(b)

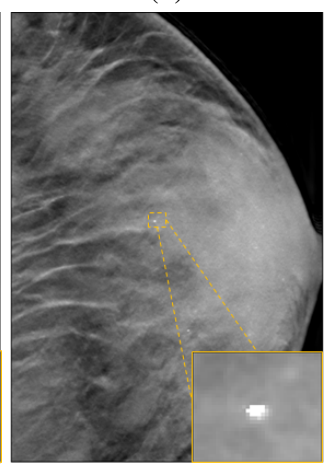

(d)
Fig. 3: Sample of slices of a DBT reconstruction with size $2344 \times$ $868 \times 44$ (pixels) : (a) Constructed $\left(\lambda_{i}\right)_{1 \leq i \leq m}$ from the representative clinical data. (b) DBT reconstruction with classical TV regularization and without the clinical term, (c) DBT reconstruction with classical TV regularization and the clinical term, (d) DBT reconstruction with SATV regularization and the clinical term.

the same breast texture quality, after fine-tuning of the regularization parameters $\eta$ and $\beta$ for this purpose. Here, $\beta=$ $600, \epsilon=10$ for classical TV and $\beta=660, \epsilon=10, \eta=0.09$ when SATV penalization is used.

\section{CONCLUSION}

Despite the tremendous amount of research works highlighting the success of TV in various image processing applications, this penalty does not model properly the heterogeneity within images. In this paper, our main contribution lies in a novel definition of a spatially adaptive total variation which incorporates prior shape knowledge on the image to be restored. In the DBT context, the proposed SATV allows to take into account the expected location of small microcalcifications. We have demonstrated on different datasets the interest of our proposed approach with respect to classical TVbased DBT reconstruction. One future direction for further improvements is to investigate techniques for a more automatic setting of the different parameters involved in SATV, depending on the targeted task and images to be processed. 


\section{REFERENCES}

[1] P. Pattacini, A. Nitrosi, G.R. Paolo, L. Valentina, V. Ginocchi, S. Ravaioli, R. Vacondio, L. Braglia, S. Cavuto, C. Campari, and the RETomo Working Group, "Digital mammography versus digital mammography plus tomosynthesis for breast cancer screening: the reggio emilia tomosynthesis randomized trial," Radiology, vol. 288, no. 2, pp. 375-385, 2018.

[2] I. Sechopoulos, "A review of breast tomosynthesis. Part I. The image acquisition process," Medical Physics, vol. 40, no. 1, pp. 014301, 2013.

[3] I. Sechopoulos, "A review of breast tomosynthesis. Part II. Image reconstruction, processing and analysis, and advanced applications," Medical Physics, vol. 40, pp. 014302, 2013.

[4] E.Y. Sidky, I.S. Reiser, R. Nishikawa, and X. Pan, "Image reconstruction in digital breast tomosynthesis by total variation minimization," in Proc. SPIE Medical Imaging, San Diego, CA, March 2007, vol. 6510.

[5] E.Y. Sidky, X. Pan, I.S. Reiser, R.M. Nishikawa, R.H. Moore, and D.B. Kopans, "Enhanced imaging of microcalcifications in digital breast tomosynthesis through improved imagereconstruction algorithms," Medical Physics, vol. 36, no. 11, pp. 4920-4932, 2009.

[6] E. Metin, Y. Isa, K. Mustafa, and A. Aydin, "An iterative tomosynthesis reconstruction using total variation combined with non-local means filtering," BioMedical Engineering OnLine, vol. 13, no. 1, pp. 65, May 2014.

[7] L. Condat, "Discrete total variation: New definition and minimization," SIAM Journal on Imaging Sciences, vol. 10, no. 3, pp. 1258-1290, 2017.

[8] R. Abergel and L. Moisan, "The shannon total variation," Journal of Mathematical Imaging and Vision, vol. 59, no. 2, pp. 341-370, 2017.

[9] A. Chambolle, S.E. Levine, and B.J. Lucier, "An upwind finitedifference method for total variation-based image smoothing," SIAM Journal on Imaging Sciences, vol. 4, no. 1, pp. 277-299, 2011.

[10] M. Nikolova, "Local strong homogeneity of a regularized estimator," SIAM Journal on Applied Mathematics, vol. 61, no. 2, pp. 633-658, 2000.

[11] J.F. Aujol, "Some first-order algorithms for total variation based image restoration," Journal of Mathematical Imaging and Vision, vol. 34, no. 3, pp. 307-327, 2009.

[12] P. Blomgren, T. F. Chan, P. Mulet, and C.K. Wong, "Total variation image restoration: numerical methods and extensions," in Proceedings of International Conference on Image Processing. IEEE, 1997, vol. 3, pp. 384-387.

[13] T. Chan, A. Marquina, and P. Mulet, "High-order total variation-based image restoration," SIAM Journal on Scientific Computing, vol. 22, no. 2, pp. 503-516, 2000.

[14] T.F. Chan, S. Esedoglu, and F.E. Park, "Image decomposition combining staircase reduction and texture extraction," Journal of Visual Communication and Image Representation, vol. 18, no. 6, pp. 464-486, 2007.
[15] G. Chierchia, N. Pustelnik, B. Pesquet-Popescu, and J.C. Pesquet, "A nonlocal structure tensor-based approach for multicomponent image recovery problems," IEEE Transactions on Image Processing, vol. 23, no. 12, pp. 5531-5544, 2014.

[16] C. Louchet and L. Moisan, “Total variation denoising using posterior expectation," in 2008 16th European Signal Processing Conference. IEEE, 2008, pp. 1-5.

[17] F. Luo, W. Li, W. Tu, and W. Wu, "Adaptive weighted total variation minimization based alternating direction method of multipliers for limited angle ct reconstruction," IEEE Access, vol. 6, pp. 64225-64236, 2018.

[18] O. Merveille, O. Miraucourt, S. Salmon, N. Passat, and H. Talbot, "A variational model for thin structure segmentation based on a directional regularization," in 2016 IEEE International Conference on Image Processing (ICIP). IEEE, 2016, pp. 4324-4328.

[19] F. Liao and S. Shao, "An image denoising fast algorithm for weighted total variation," in Proceedings of the 2 nd International Conference on Intelligent Information Processing. ACM, 2017, p. 16.

[20] G. Xu, Y. Xia, and H. Ji, "Weighted total variation based convex clustering," arXiv preprint arXiv:1808.09144, 2018.

[21] S. Biton and G. Gilboa, "Adaptive anisotropic total variation-a nonlinear spectral analysis," arXiv preprint arXiv:1811.11281, 2018.

[22] M. Sghaier, E. Chouzenoux, G. Palma, J.C. Pesquet, and S. Muller, "A new approach for microcalcification enhancement in digital breast tomosynthesis reconstruction," in 2019 IEEE 16th International Symposium on Biomedical Imaging (ISBI 2019). IEEE, 2019, pp. 1450-1454.

[23] I. Christoyianni, A. Koutras, E. Dermatas, and G. Kokkinakis, "Computer aided diagnosis of breast cancer in digitized mammograms," Computerized Medical Imaging and Graphics, vol. 26, no. 5, pp. $309-319,2002$.

[24] P.L. Combettes and J.C. Pesquet, "Proximal splitting methods in signal processing," in Fixed-point algorithms for inverse problems in science and engineering, pp. 185-212. Springer, 2011.

[25] S. Boyd, N. Parikh, E. Chu, B. Peleato, and J. Eckstein, "Distributed optimization and statistical learning via the alternating direction method of multipliers," Foundations and Trends® in Machine learning, vol. 3, no. 1, pp. 1-122, 2011.

[26] N. Komodakis and J.C. Pesquet, "Playing with duality: An overview of recent primal? dual approaches for solving largescale optimization problems," IEEE Signal Processing Magazine, vol. 32, no. 6, pp. 31-54, 2015.

[27] American College of Radiology et al., "Acr bi-rads atlas: breast imaging reporting and data system," Reston, VA: American College of Radiology, vol. 2014, pp. 37-78, 2013. 